\title{
FACTORES PARA IMPLEMENTAR LA RESPONSABILIDAD SOCIAL UNIVERSITARIA (RSU) EN INSTITUCIONES DE EDUCACIÓN SUPERIOR PÚBLICAS
}

\author{
Francisco Gerardo Barroso Tanoira*, Raúl Alberto Santos Valencia**, Jorge Iván Ávila Ortega**, \\ Ma. Eugenia López Ponce**, José Rubén Bacab Sánchez ${ }^{\star \star}$
}

\begin{abstract}
Barroso-Tanoira F.B., Santos-Valencia R.A., ÁvilaOrtega J.I., López-Ponce M.E., Bacab-Sánchez J.R. Factores para implementar la Responsabilidad Social Universitaria (RSU) en Instituciones de Educación Superior. Hitos de Ciencias Económico Administrativas 2015;21 (59): 7-18
\end{abstract}

\section{RESUMEN}

Objetivo: Identificar las causas por las que la implementación de esfuerzos de RSU no son eficaces en universidades públicas, así como proponer estrategias para lograr un comportamiento socialmente responsable en dichas instituciones.

Material y método: El estudio consistió en dos fases; en la primera, tomó parte quien dirige la Facultad de la universidad participante; $y$ en la segunda, participaron alumnos y administrativos de la misma. A nivel Dirección, se administró una guía de entrevista, al igual que para los administrativos, pero para los alumnos se utilizó un cuestionario ya validado. Se usaron criterios para interpretar la frecuencia de mención de las respuestas cualitativas, así como escalas para las cuantitativas.

Resultados: El mayor puntaje promedio corresponde a calidad de vida laboral, seguida por la relación con los usuarios, cuidado y preservación del medio ambiente y la relación con la comunidad. Desde la percepción del alumno, la institución se preocupa más por mejorar la calidad de vida de su personal y la relación con los usuarios, pero falta involucrarse en acciones efectivas de cuidado al medio ambiente y que mejoren la relación y vinculación con la comunidad.
Barroso-Tanoira F.B., Santos-Valencia R.A., ÁvilaOrtega J.I., López-Ponce M.E., Bacab-Sánchez J.R. Factors to implement University Social Responsibility (USR) in public higher education institutions. Hitos de Ciencias Económico Administrativas 2015;21 (59): 7-18

\section{ABSTRACT}

Objective: Identify the reasons why the efforts on implementing university social responsibility (USR) in public universities have not been effective, as well as propose strategies to achieve a socially responsible behavior in such institutions.

Material and method: The study consisted on two stages; in the first one, the Faculty Director participated; and in the second one, the participants were students and administrative workers. An interview guide was administered to the Director and the administrative workers, and an already validated questionnaire was applied to the students. Criteria for interpreting the frequency of qualitative responses were used, as well as scales for quantitative ones.

Results: The highest average score corresponds to quality of working life, followed by the relationship with users, care and preservation of the environment and the relationship with the community. From the perception of the students, the institution is more concerned about improving the quality of life of its employees and the relationship with users, but lacks involving in effective actions in caring for the environment as well as the improvement of the relationship and community linkage.

\footnotetext{
* Profesor investigador en la Universidad Anáhuac Mayab, División de Negocios. Doctor en Ciencias Administrativas por la Universidad Anáhuac Mayab.

** Profesor de Tiempo Completo adscrito al Programa Educativo de Ingeniería Industrial del Instituto Tecnológico Superior de Calkiní en el Estado de Campeche. Campeche (ITESCAM). Calkiní, Campeche, México.
} 
Conclusiones: Los factores para implementar exitosamente la RSU, son el compromiso y participación del personal de la institución, las acciones conjuntas con otras instituciones, énfasis en el cuidado ambiental, participación de la institución en actividades sociales, procurar la satisfacción de los usuarios de la Institución, apreciar la importancia de la institución para el desarrollo, así como mantener y mejorar las relaciones con el gobierno. Sin embargo, también deben considerarse la habilidad para negociar de las autoridades con los profesores, sindicato y alumnos, así como promover los valores, visión y misión de la institución.
Conclusions: The factors to implement successfully the USR are commitment and participation of the staff of the institution; the joint actions with other institutions; the emphasis on the environmental care; the participation of the institution in social activities; the satisfaction of the institution users, the fact of appreciating the importance of the institution for the development, as well as keeping and improving the relationships with the government. However, it is also necessary to consider the ability of the authorities to negotiate with the professors, union and students, as well as to promote the institutional values, vision and mission.
Palabras clave: Responsabilidad social empresarial. Responsabilidad social universitaria. Instituciones de Educación Superior. Compromiso social. Universidades públicas.
Key words: Corporate social responsibility. University social responsibility. Higher Education Institutions. Social commitment. Public universities.

DIRECCIÓN PARA RECIBIR CORRESPONDENCIA: Correo electrónico: francisco.barroso@anahuac.mx y fbarroso_tanoira@yahoo.com.mx

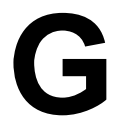

eneralidades de la Responsabilidad Social (RS)

Ante las demandas y rápidos cambios del mundo actual, las organizaciones deben adaptarse a su medio lo más rápidamente posible, pero de manera sostenible. Sus directivos están conscientes de que sus acciones y operaciones tienen un profundo impacto en la sociedad que les rodea, para bien o para mal, y de que hay que hacer algo para construir una sociedad mejor y más sustentable (Rochlin, 2005). Es claro que el objetivo no es maximizar las utilidades de los accionistas a costa del trabajador, del deterioro del medio ambiente, la falta de ética en el trato a clientes, proveedores, accionistas y gobierno, así como la falta de cooperación con el desarrollo del lugar donde la organización opera.

Para Porto y Castromán (2006), es cierto que no se ha logrado una definición de RS comúnmente aceptada, pues existe aún confusión sobre su alcance real. Como indica Rochlin (2005), hay quienes la confunden con filantropía o con actos de buena voluntad, como construir escuelas en los lugares donde la organización opera, sea una empresa, dependencia de gobierno, iglesia u organización de la sociedad civil. Sin embargo, gradualmente se va refinando este concepto y se resalta su carácter de iniciativas voluntarias en torno a tres aspectos: el medioambiental, el laboral y la ética del comportamiento organizacional con los diversos grupos con los que se relacionan las organizaciones. Dicho autor sostiene que las organizaciones deben ir más allá del enfoque inmediatista de solo cumplir con las normas establecidas, pues no basta con cumplir con ellas para ser socialmente responsable. Sería como aceptar que lo legal es siempre lo justo. Tampoco es una moda, sino que se trata de alinear el quehacer económico con el social y medioambiental (Rochlin, 2005). Se espera que las medidas que adopten mitiguen los impactos ambientales sin afectar las utilidades de sus accionistas, generando un aumento en el valor de la organización. Se trata de que sean capaces de enfrentar los embates de la globalización a través del mejoramiento de su personal y del trato ético con todos los involucrados en el negocio más allá de las utilidades. Sin embargo, para las organizaciones frecuentemente la RSE es sinónimo de mejoramiento del clima laboral, comercialización o de captación de clientes, lo cual hace que tenga para ellas un fin práctico, a manera de herramienta, en lugar de ser una filosofía de trabajo (Barroso, 2008).

\section{Concepto de RSE}

Puede conceptualizarse la RSE como la integración voluntaria por parte de las organizaciones, de las preocupaciones sociales y medioambientales en sus operaciones comerciales y con sus interlocutores. También puede definirse como la contribución activa y 
voluntaria de las organizaciones en el mejoramiento social, económico y ambiental (Libro Verde de la Unión Europea, 2001, citado por Porto y Castromán, 2006; Barroso, 2008). Bajo este concepto de administración, se engloba un conjunto de prácticas, estrategias y sistemas de gestión, que persiguen un nuevo equilibrio entre las dimensiones económica, social y ambiental; ya que ser socialmente responsable no significa solamente acatar las obligaciones jurídicas plenamente, sino que es ir más allá de su cumplimiento invirtiendo más en el capital humano, en el entorno y en las relaciones con los interlocutores.

El enfoque al bien común debe llevarla a las organizaciones a crear riqueza de manera justa y eficiente, respetando la dignidad y los derechos inalienables de los individuos (Ramos, 2006), lo que implica evitar actos de corrupción en el gobierno corporativo y en las operaciones de toda la organización, así como implementar sanciones si ocurrieran. Por su parte, Medina (2006) sostiene que la RSE debe ser parte de la esencia de la organización y estar inscrita en la misión y visión de la misma, implicando un cambio integral e invirtiendo para ello los recursos y esfuerzos necesarios para realizar un proyecto de renovación de la imagen corporativa por convencimiento propio, permitiendo la participación activa de los involucrados debido a que se trata de la búsqueda de la calidad en todas sus acciones. Desde este punto de vista, ser socialmente responsable no significa limitarse a acatar las obligaciones jurídicas, sino que es ir más allá de su cumplimiento, invirtiendo más en el capital humano, en el entorno y en las relaciones con los interlocutores (Rochlin, 2005).

\section{¿Qué es una Organización Socialmente Responsable?}

Para Porto y Castromán (2006), la RSE es la conciencia del comportamiento y la acción de mejora continua, medida y consistente, que hace posible a la organización ser más competitiva, cumpliendo con las expectativas de todos sus participantes o stakeholders en lo particular, y de la sociedad en general, respetando la dignidad de la persona y de las comunidades en que opera, así como su entorno (Sánchez, Placencia y Pedroza, 2007). Por lo tanto, las que se comprometan con estas prácticas pero que no tengan el marco adecuado pueden llegar a creer que dicho compromiso es simple apariencia. Cabe mencionar que los stakeholders son las personas o grupos que tienen propiedad, derechos o intereses en una organización y en sus actividades, pasadas, presentes o futuras, las cuales pueden ser legales 0 morales, individuales o colectivas (Rojas, M'Zail, Torcotte y Kooli, 2006).

\section{Requisitos para ser una Organización Socialmente Responsable}

Curiosamente, aunque la RS no es filantropía, es el Centro Mexicano para la Filantropía (CEMEFI), organismo fundado en 1988, una de las entidades que evalúa a las organizaciones en cuanto a RS. En sus revisiones incluye cuatro ámbitos que debe cumplir toda empresa $u$ organización socialmente responsable, y mediante los cuales las certifica (Porto y Castromán, 2006; Barroso, 2008): (1) contribuir a la calidad de vida dentro de la organización; (2) cuidado y preservación del medio ambiente; (3) desempeñarse con un código de ética, y (4) vincularse con la comunidad a partir de la misión del negocio, pero también de los bienes y servicios producidos. Las Organizaciones Socialmente Responsables, en el sentido amplio del concepto, deben cumplir con los cuatro ámbitos que propone el CEMEFI al mismo tiempo (Barroso, 2008). Si practican algunos de ellos, entonces sólo estarán realizando acciones sociales o de compromiso organizacional con la sociedad, pero no puede decirse que sean socialmente responsables en el sentido completo del concepto. En la RS interviene el liderazgo como la intención de sumar a otros, en que el jefe, director o responsable debe tener la capacidad para conducir a su organización para enfrentar eficazmente los problemas económicos, sociales y ambientales que se presenten (Rochlin, 2005; Barroso, 2008). Es necesario incorporar la RS en el corazón de la organización, pero ésta comienza desde uno mismo y luego se va extendiendo en la estructura y las personas que la componen.

\section{Responsabilidad Social Universitaria (RSU)}

La RS en IES puede denominarse RESIES, de manera general. Sin embargo, al hablarse específicamente de universidades, como se abordará en este trabajo, se usará el término Responsabilidad Social Universitaria o RSU, tal como sugieren Vallaeys, De la Cruz y Sasia (2009), el cual es un concepto que se escucha cada vez más en el ámbito de la educación superior y en discusiones sobre ciudadanía, sostenibilidad y servicio a la comunidad. 
Para entender la RSU cabe evocar otro concepto similar que le precede: la Responsabilidad Social Empresarial (RSE). Dado que las universidades son también organizaciones que tienen impactos de diversa naturaleza en la sociedad y comunidades, la reflexión sobre la RSE se traslada también al mundo universitario y de las IES en general. Por ello, desde su misión académica, las universidades deben promover una mayor orientación e involucramiento hacia las necesidades de la sociedad, por lo que la RSU se puede concebir como el compromiso activo y voluntario que tienen las universidades para difundir y poner en práctica un conjunto de principios y valores generales y específicos que les permitan orientar su desempeño educacional, económico y social para desempeñar adecuadamente sus funciones de docencia, investigación y extensión del conocimiento a través del mejoramiento de la calidad de vida de su personal, preservando el medio ambiente, fomentando trato ético hacia todos sus stakeholders y procurando el desarrollo local y regional. Entonces, el comportamiento socialmente responsable de las universidades no es sólo una acción externa o un punto a cumplir para lograr una acreditación, sino que tiene incidencia académica a través de la introducción de cambios en el currículo, en la pedagogía y en la definición de sus líneas de investigación. La RS debe comenzar desde adentro, tal y como recomienda Somoggi (2005).

\section{Formas de la RSU}

Existen diferentes formas en las que una universidad o cualquier IES puede ayudar a la sociedad, las cuales se clasifican en cuatro tipos (Valverde, 2011):

\section{Promoción de impactos positivos en el entorno} inmediato. Se refiere a la creación de centros universitarios o de educación superior al servicio de la comunidad, como clínicas y proyectos productivos para desarrollo comunitario.

\section{Promoción de un impacto comunitario: Son} actividades a realizar en instalaciones de las universidades para atender una necesidad del colectivo.

3. Actividades de asistencia técnica. Asesorías o consultoría y actividades de extensión realizadas por la universidad para beneficio de la comunidad, como bufetes jurídicos y consultoría empresarial.

4. Actividades para el fortalecimiento de las instituciones mediante el voluntariado. La universidad se vincula a instituciones en las que alumnos pueden ayudar de forma voluntaria.

La RSU, puede entenderse como el establecimiento de cuatro ejes (Vallaeys et al., 2009):

1) Campus responsable. Las universidades deben operar con un enfoque de gestión socialmente responsable de la organización y sus procedimientos institucionales, poniendo mayor énfasis en el respeto de los derechos humanos, equidad, no discriminación, atención al desarrollo personal, profesional y en la institución. Abarca el respeto de los derechos laborales, buen clima y satisfacción laboral, cuidado del medio ambiente, transparencia y rendición de cuentas, selección de proveedores con criterios sociales y ambientales, procesos participativos, cumplimiento de la legalidad, comportamiento ético y promoción de la RSU entre los directivos, docentes, administrativos y alumnos.

2) Formación profesional y ciudadana. Se refiere a promover y realizar un trabajo como institución educativa dirigido a los siguientes componentes: presencia de temáticas ciudadanas y de responsabilidad social en el currículo (derechos humanos, desarrollo sustentable, ética profesional y cívica, gestión de la responsabilidad social, etc.). Incluye la articulación entre: (1) profesionalización y voluntariado solidario; (2) aprendizaje profesional basado en proyectos sociales; (3) comunidades de aprendizaje; (4) integración de actores sociales externos en el diseño de los mapas curriculares; (5) modelo educativo orientado al aprendizaje y con pertinencia social; (6) estudios de oferta y demanda de los programas educativos, de empleadores y seguimiento de egresados; (7) equidad en el acceso a la formación; (8) evaluación del cumplimiento del perfil de egreso, y (9) dimensión internacional en los programas educativos.

3) Gestión social del conocimiento. Una universidad socialmente responsable integra actores sociales externos en el diseño de las líneas de investigación y su participación en los proyectos. Difunde y 
transfiere el conocimiento socialmente útil a la sociedad, en lo general y en lo particular, a la población desfavorecida. Posee pertinencia social de la investigación, en particular en su articulación con la agenda local y regional del desarrollo, promoviendo investigaciones aplicadas a temas del desarrollo, ampliación de las oportunidades de acceso al conocimiento y vinculación de la investigación con la formación profesional y ciudadana.

4) Participación social. El fin de una IES, en este caso una universidad, no es generar el mayor número de egresados, sino que éstos se integren a la promoción de la autogestión y autonomía en las acciones del desarrollo comunitario, promoción de redes sociales para el desarrollo, participación activa en la agenda local y nacional del desarrollo, así como en proyectos sociales con participación de estudiantes y académicos con apoyo institucional, formación de redes universitarias de RSU y participación en la formulación de políticas públicas para el desarrollo.

Para Chomsky (2003), lamentablemente las universidades, y las IES en general, son «económicamente parasitarias» porque dependen del apoyo externo para poder desarrollar sus principales funciones, lo que las obliga a preocuparse en exceso por la obtención de recursos económicos de fuentes externas, tanto públicas como privadas, para poder funcionar. Sin embargo, este esfuerzo les lleva a desatender un papel más crítico y propositivo para mejorar las carencias y ambigüedades del actual sistema económico, político y social, lo que crea un dilema para las IES entre cumplir con su responsabilidad social de ejercer una postura liberadora y subversiva, o ir en contra de los intereses de quienes las financian, ya sean empresas o el mismo Gobierno. Por su parte, Kliksberg (2009) sostiene que las universidades deben asumir un papel de liderazgo en políticas concretas tales como: (1) la formación ética de los jóvenes que asisten a la universidad; (2) dar prioridad, en cuanto a esfuerzos de investigación, a las temáticas que representen los problemas más apremiantes de la población; (3) participar activamente en el debate de los grandes temas sociales; (4) extender la labor educativa a la sociedad en su conjunto y no sólo de sus estudiantes, orientándose especialmente hacia los sectores más desfavorecidos, y (5) promover y apoyar el potencial de voluntariado de los jóvenes que ingresan a la universidad.

Según Vallaeys et al. (2009), la RSU toma en cuenta los impactos que la universidad genera en su entorno; (1) organizacionales; (2) educativos; (3) cognitivos, y (4) sociales. Los impactos organizacionales y sociales son comunes para cualquier organización. Sin embargo, los que diferencian a las universidades de las empresas son los educativos y cognitivos, especialmente estos últimos, ya que definen los paradigmas mentales y modelos prácticos a través de los cuales la institución se aproxima a las necesidades de la sociedad, así como los modos de comprender la realidad. No se trata sólo de insistir en las tres funciones sustantivas que son la docencia, la investigación y la extensión, y de confiar a esta última la tarea de vincular a las IES con la sociedad. Son los cuatro procesos - gestión, formación, producción de conocimientos y participación sociallos que constituyen la función social de la universidad, pero son estériles si no se fortalecen aspectos interdisciplinarios, el pensamiento crítico, el emprendimiento y la ciudadanía activa.

Para De la Cruz (2011), las universidades e IES en general no están comprometidas únicamente con responder a las demandas de la sociedad, y mucho menos hacerlo parcialmente o solamente algunas de ellas. El riesgo y la limitación es que exista una orientación hacia lo económico, olvidando que el ser humano es el centro de la misión universitaria.

\section{Planteamiento del problema}

Pese a que se indique que se practica la RSU, no se están abarcando los cuatro ámbitos del CEMEFI citados por Porto y Castromán (2006) y que, según Barroso (2008), debe practicar toda organización al mismo tiempo para ser socialmente responsable en el sentido completo del concepto, y que son requeridos también en el concepto de RSU expuesto en este trabajo. Según las iniciativas presentadas, se piensa que con incrementar becas, ampliar cobertura, crear departamentos para protección de flora y fauna, así como con realizar proyectos sociales es suficiente para decir que se practica la RSU. Se da más atención al campus responsable, a la formación profesional, a la gestión social del conocimiento o a la participación 
social que abarcan los ejes propuestos por Vallaeys et al. (2009), pero no hay indicios de que se realice al mismo tiempo o que existan esfuerzos para que estas actividades permanezcan de manera sostenible. Esto se ve ratificado por un estudio realizado por Collí y Cano (2014) en una universidad del Sureste de México con un instrumento diseñado con base en dichos ejes y sus impactos. Los resultados arrojan que es necesario implementar un programa de RSU que abarque integralmente los cuatro ámbitos del CEMEFI citados por Porto y Castromán (2006) y Barroso (2008) con el que estén todos los niveles comprometidos, desde la Rectoría hasta los grupos de interés internos y externos de la institución.

Entonces, aparentemente se están realizando acciones de compromiso social en lugar de ser socialmente responsables en el sentido amplio del concepto. ¿Por qué sucede esto? ¿Cómo puede fomentarse la RSU? ¿Qué impide a las IES ser socialmente responsables? Para ejemplificar esto se presentará un estudio realizado en una Facultad de una universidad pública en el sureste de México. Las autoridades de dicha Facultad indican que se promueve en ella la práctica de la RSU, pero que no se sabe si sus esfuerzos son eficaces para ello. Los resultados son válidos únicamente para la Facultad en la que se realizó el estudio, pero la metodología sí puede utilizarse en otros contextos. Por lo tanto, el objetivo del presente trabajo es identificar las causas por las que la implementación de esfuerzos de RSU no son eficaces, así como proponer estrategias para lograr un comportamiento socialmente responsable.

\section{Justificación}

La identificación de las causas por las que no funcionan los esfuerzos de RSU en la universidad participante podrían ser comunes a otras IES en la región o en todo el país, por lo que identificarlas puede ayudarla a proponer planes de mejoramiento del comportamiento socialmente responsable, en vías a la sostenibilidad. Los hallazgos pueden incorporarse también a los planes de estudio en las materias de desarrollo de emprendedores, responsabilidad social o afines. Con esto la universidad cumple con los esfuerzos e impactos sugeridos por Vallaeys et al., (2009) y De la Cruz $(2010,2011)$. Además, la metodología seguida puede ser útil para el monitoreo y seguimiento de las acciones, de manera que la práctica de la RSU sea una filosofía en las instituciones educativas.

\section{MATERIAL Y MÉTODO}

\section{Tipo y diseño del estudio}

El presente estudio consta de dos fases, una cualitativa y otra cuantitativa. En la primera, se realizó una entrevista a la máxima autoridad de la Facultad con el objetivo de obtener información sobre las acciones que se llevan al cabo en materia de RSU, así como en los resultados desde su percepción. En la segunda, se obtuvo información sobre el estado de la RSU en la Facultad, desde la percepción administrativos y alumnos.

En la fase 1, el estudio fue exploratorio y descriptivo, con diseño no experimental, ya que los investigadores no tuvieron injerencia en la producción de las respuestas, limitándose a registrarlas. Además, fue transversal porque se efectuó en un solo momento sin seguimiento (Hernández, Fernández y Baptista, 2010). El método fue el estudio de campo y la técnica fue la entrevista, la cual fue de tipo semiestructurada. Posteriormente, en la fase 2, el estudio fue de tipo exploratorio y descriptivo, con diseño no experimental transversal; el método fue el trabajo de campo y la técnica la encuesta, utilizando cuestionarios como instrumentos. Esta fase consistió en dos partes: participación de alumnos y de los administrativos que estaban disponibles en el momento del estudio.

\section{Participantes en el estudio}

En la fase 1, solamente participó quien dirige la Facultad. Se determinó considerar su intervención como una fase debido a la relevancia de su testimonio en cuanto a acciones y comportamiento de la Facultad a su cargo, en cuanto a RSU. En la primera parte de la fase 2 participaron los 30 alumnos (16 mujeres) que estaban disponibles en el momento del estudio, distribuidos en dos salones de clase, más algunos que estaban en diferentes partes de las instalaciones. Cabe mencionar, que el momento del trabajo de campo fue realizado a sugerencia de quien dirige la Facultad. De los alumnos, 28 de ellos son de $4^{\circ}$ a $8^{\circ}$ semestre de licenciatura, pero dos son de maestría (ambos varones). Se decidió incluirlos porque han estado estudiando por más de un año con la institución. En esta fase también se recabó la percepción de los tres administrativos presentes en 
ese momento, cuyo testimonio se consideró importante debido a que una es la asistente de la Dirección, uno es el Coordinador Administrativo y el último, es el Coordinador Académico.

\section{Instrumentos y procedimientos}

En la fase 1, se utilizó una guía de entrevista semiestructurada para determinar las acciones que realiza en materia de RSU, así como su percepción sobre los resultados y la participación de su personal. La validez se determinó por el juicio de tres expertos (Hernández et al., 2010). Por otra parte, en el cuestionario para alumnos de la fase 2 , se incluyeron reactivos con escala de Likert para verificar la participación de alumnos y personal de la Facultad, desde la percepción de los mismos alumnos y según los cuatro ámbitos del CEMEFI, citados por Barroso (2008) y Porto y Castromán (2006), pero adaptados a la naturaleza de la institución educativa según los lineamientos de la RSU de Valverde (2011) y los ejes de Vallaeys et al. (2009). La validez de contenido y de criterio se determinó por el juicio de tres expertos relacionados con acciones de RSE en empresas y en IES públicas (Hernández et al, 2010), y la confiabilidad mediante la administración de dicho cuestionario a tres alumnos del tipo de los que participarían para verificar tendencia de respuestas, lo cual evidenció la consistencia de los patrones de respuesta, por lo que se consideró confiable. Al final del trabajo de campo se calculó el coeficiente alfa de Cronbach a manera de estudio comprobatorio, obteniendo 0.936 , lo que ratificó su confiabilidad. Además, se realizó un análisis factorial comprobatorio al final del estudio para verificar su validez de constructo. Cabe mencionar, que el cuestionario para personal administrativo se elaboró de manera similar al de los alumnos, pero con preguntas ad hoc para el personal de la Facultad; solo fue sometido a validez de contenido y constructo por el juicio de los mismos expertos que para el cuestionario de alumnos, resultando favorable.

Previos permisos y acordadas fecha y hora, se administraron los cuestionarios a los participantes por los investigadores y un grupo de cinco becarios capacitados para el efecto. Se les explicó el por qué del cuestionario a los alumnos participantes, quienes procedieron a llenarlos, tardando alrededor de 15 minutos cada uno. Para los administrativos fue un procedimiento idéntico, pero administrados por integrantes del grupo investigador, no por los becarios. Al concluir, se procedió a la entrevista con la Directora, la cual duró cerca de 60 minutos.

\section{Plan de análisis de la información}

Los resultados cuantitativos se interpretaron de la siguiente manera: de 1 a 1.49 (Totalmente en desacuerdo); de 1.5 a 2.49 (En desacuerdo); de 2.5 a 3.49 (Más o menos de acuerdo); de 3.5 a 4.49 (De acuerdo), y de 4.5 a 5 (Totalmente de acuerdo). Para la información cualitativa se agruparon las respuestas repetidas (frases testimonio) por frecuencia de mención, asignándoles ideas clave representativas y agrupando dichas ideas en categorías, según la tabla 1.

\section{Tabla 1}

Frecuencia de las ideas clave (Savall, 2005)

\begin{tabular}{ll} 
Cuantitativa & Cualitativa \\
\hline Más del $90 \%$ de las personas & Siempre \\
Del $70 \%$ a $89 \%$ de las personas & Muy frecuentemente \\
Del $50 \%$ a $69 \%$ de las personas & Frecuentemente \\
Del $30 \%$ a $49 \%$ de las personas & Varias veces \\
Del $11 \%$ a $29 \%$ de las personas & Pocas veces \\
De 1 persona a $10 \%$ & Rara vez \\
\hline
\end{tabular}




\section{RESULTADOS}

\section{Fase 1. Resultados cualitativos (con porcentaje de menciones)}

Se entiende como RSU el hacer responsables a los administrativos y alumnos, promoviendo temas que sean de impacto para la sociedad, buscando la armonía social, la sana interacción y el beneficio social económico. De manera general, aunque la Dirección y los administrativos coinciden en la existencia de programas para fomentar el cuidado al medio ambiente y el apoyo a la comunidad en general, los alumnos no perciben que exista realmente un compromiso de la universidad con su entorno. Por cierto, éstos frecuentemente demostraron falta de conocimiento sobre las acciones de RSU que la Dirección mencionó (33.3\%), además de que no saben a qué se refiere concretamente el término de RS, cosa que los llevó a confundirse en varias ocasiones al tratar de definir el concepto.

La participación de la Facultad ha sido diseñar e implementar acciones de RSU, estrategias de reciclaje, sistemas de gestión ambiental, anticorrupción, salud y prevención de adicciones. Se ha procurado que los alumnos se involucren en proyectos comunitarios en que se apoye llevando juguetes, artículos de uso personal y visita a albergues, llevando a la gente necesitada clases de lectura, cobijas y otros satisfactores. También se realizan brigadas de salud. Sin embargo, actualmente no se involucran en las actividades, por lo que se percibe cierta apatía en ellos para participar en acciones de RSU (26.7\%). Es probable que eso se deba al desinterés que tiene la juventud con respecto a dicho tema. Desde luego, en cuanto a los profesores de la Facultad, algunos si están interesados y otros son indiferentes.

Hay algunos alumnos destacados en el ámbito cultural. También se tiene un albergue especial para los alumnos de escasos recursos, además de que a algunos estudiantes se les han otorgado becas. También se han implementado actividades de apoyo a la comunidad $(26.7 \%)$, como en el caso de la Facultad de Medicina, que ha realizado sesiones de risoterapia y reparto de cobijas y medicinas.

La Facultad ha obtenido un premio de RSU a nivel nacional por la lengua indígena, gracias a un alumno que habla la lengua maya e imparte clases a las comunidades. Sin embargo, si la Facultad tuviera las posibilidades suficientes para realizar diversas acciones, se llevarían a cabo las de apoyo para proyectos a las personas vulnerables de escasos recursos, así como impartir diversos cursos a la sociedad.

Todos en la Facultad coinciden en la necesidad de crear proyectos que apoyen a la comunidad en general, que signifiquen acciones dirigidas a la salud y seguridad de la sociedad. Cabe mencionar, que los alumnos con poca frecuencia sabían que responder (26.6\%), se les notaba confusos y las respuestas que daban encajaban más en el marco de la filantropía (10\%). Sin embargo, llama la atención el que algunos alumnos hayan respondido que la Facultad no pueda involucrarse en acciones de RSU (6.7\%).

\section{Acciones realizadas en cuanto al cuidado al medio ambiente}

Dirección y administrativos mencionaron que se han realizado campañas de reforestación promoviendo la responsabilidad del alumnado, cursos de desarrollo de cultura y cuidado de energía, cursos de inducción de «salud reproductiva», eventos y cursos de SEMARNAT, transparencia pública, servicio y apoyo a la comunidad. Se han otorgado recursos para proyectos productivos brindando asesorías gratuitas y la campaña «Huella ecológica».

Para los alumnos, el cuidado al ambiente se remite a clasificación y reciclaje de basura (33.3\%). Sin embargo, Ilama la atención el que la segunda respuesta fuera el que no saben si la Facultad realiza acciones de cuidado ambiental (20\%) y que el $3.3 \%$ dijera que no se realizan acciones conservacionistas en absoluto. Aunque la Facultad sí tenga en desarrollo programas de RSU, no se les da la adecuada difusión entre el personal y alumnos (20\%), dejando de lado las intenciones de participar que puedan tener algunos de los integrantes. Otros comentarios se dirigieron a que la Universidad no se involucra con el cuidado y preservación del medio (3.3\%) y a que los profesores enseñen de manera más práctica (3.3\%), así como pidieron un trato más amable por parte del personal de la Facultad (3\%). Debe promoverse la RSU con los alumnos y organizar campañas de diferentes tipos, favoreciendo la cultura $y$ el apoyo a grupos vulnerables social y económicamente (33.3\%). 
Fase 2. Resultados cuantitativos (con el promedio de los puntajes según la escala)

\section{Calidad de vida laboral}

Los alumnos están de acuerdo en que sus profesores están comprometidos hacia la Facultad (3.72), que están satisfechos con sus labores (3.54) y que ésta es un buen lugar para trabajar (3.52). Sin embargo, están más o menos de acuerdo en que se les capacita (3.34) y de que están comprometidos hacia la institución (3.27), al igual que los administrativos. En resumen, están más o menos de acuerdo en que la Facultad propicia un buen ambiente laboral y general (3.42).

\section{Cuidado y preservación del medio ambiente}

Están de acuerdo (3.7) en que realmente se respeta el entorno ecológico en la operación de la Facultad, pero no perciben con seguridad que la Facultad colabore con organismos o instituciones dedicadas al cuidado ambiental (3.07) o que se participe en actividades que cuiden el medio (3.0), lo que refuerza el argumento de que falta difundir lo que se hace para preservación del entorno (3.16).

\section{Relación con la comunidad}

Los alumnos están más o menos de acuerdo en que la Facultad está realizando acciones importantes para el desarrollo de la comunidad (3.31). No hay seguridad de que se identifiquen las necesidades sociales del entorno (3.2) ni de que se involucre realmente con la comunidad. También están más o menos de acuerdo en que la Facultad promueve una cultura de RSU hacia la comunidad (3.2) y de que colabora con problemas sociales (3.1). No se percibe con certeza que haga públicos sus valores (3.07); eso sí, están más o menos de acuerdo en que la Facultad está apoyando a movimientos de caridad (2.8), pero solo de tipo filantropía, que como indica Barroso (2008), no es sinónimo de RSU.

\section{Relación con usuarios}

Los alumnos están de acuerdo (3.7) en que la Facultad se relaciona con el Gobierno y de que existe una relación ética con los stakeholders (3.52). Sin embargo, están más o menos de acuerdo (3.47) en que los servicios de la Facultad son competitivos en el mercado y de que exista vinculación activa con centros de investigación científica e instituciones educativas (3.47).
Tampoco se percibe con seguridad que haya vinculación con empresarios de la localidad (3.31) ni de que los usuarios estén satisfechos con la calidad y variedad de servicios ofrecidos por la Institución (3.03).

El mayor puntaje promedio corresponde a calidad de vida laboral (3.42), seguida por la relación con los usuarios (3.37), cuidado y preservación del medio ambiente (3.16) y finalmente, la relación con la comunidad (3.1). Entonces, desde la percepción del alumno, la institución se preocupa más por mejorar la calidad de vida de su personal y la relación con los usuarios, pero falta involucrarse en acciones efectivas de cuidado al medio ambiente y que mejoren la relación y vinculación con la comunidad.

\section{Resultados del análisis factorial}

Se realizó un análisis factorial por el método de componentes principales y rotación varimax. En él, los resultados cargaron en siete factores, cuyos nombres, resultados y varianzas explicadas aparecen en la tabla 2 y que son responsables del $81.8 \%$ de la varianza total explicada. Puede verse que el factor más crítico $y$, por lo tanto, el más importante, es el compromiso y participación del personal de la Facultad, seguido por acciones conjuntas con otras instituciones, la importancia por el cuidado ambiental, actividades para el mejoramiento de la sociedad y la procuración de la satisfacción de los usuarios con el servicio. También se debe promover en la Facultad la importancia del desarrollo comunitario y la relación con el gobierno.

\section{DISCUSIÓN}

De los resultados, puede verse que aunque las autoridades de la Facultad indican que se están realizando actividades de RSU, y de hecho hay algunas muy concretas como un programa de cuidado ambiental registrado, las acciones no son de impacto trascendente o dicho impacto no es percibido por los alumnos. Como la institución lleva al cabo acciones en los cuatro ámbitos del CEMEFI (Barroso, 2008; Porto y Castromán, 2006), pero no lo está haciendo al mismo tiempo, entonces no es socialmente responsable en el sentido amplio del concepto, sino que solo ejecuta acciones de compromiso social, que son buenas en sí, pero cuyo alcance no se percibe ni por los administrativos ni por los estudiantes. Esto se ve corroborado porque tampoco se practican al mismo tiempo los cuatro ejes de la RSU de Vallaeys et al. 


\section{Tabla 2}

\section{Resultados del análisis factorial}

\begin{tabular}{lccc} 
Nombre de la nueva variable (factor) & $\begin{array}{c}\text { Promedio } \\
\text { por factor }\end{array}$ & $\begin{array}{c}\text { Resultado } \\
\text { según la escala }\end{array}$ & $\begin{array}{c}\text { Varianza } \\
\text { explicada (\%) }\end{array}$ \\
\hline Compromiso y participación del personal de la institución & 3.47 & Más o menos & 16.8 \\
Acciones conjuntas con otras instituciones & 3.32 & Más o menos & 13.7 \\
Cuidado del medio ambiente & 3.35 & Más o menos & 12.0 \\
Actividades de mejoramiento para la sociedad & 3.10 & Más o menos & 11.2 \\
Satisfacción de los usuarios con el servicio & 2.97 & Más o menos & 10.5 \\
Importancia de la institución para el desarrollo & 3.26 & Más o menos & 9.5 \\
Relación con el gobierno & 3.76 & De acuerdo & 8.1 \\
\multicolumn{1}{c}{ Varianza explicada } & & & 81.8 \\
\hline
\end{tabular}

(2009), ya que por los resultados del estudio se aprecia que se pone más énfasis en el cumplimiento del campus responsable y la formación de personal de calidad, pero hay que practicar la gestión social del conocimiento e incrementar la participación social. También falta que la Facultad haga públicos sus valores, que dé a conocer las acciones de RSU que realiza (Valverde, 2011) y que reflexione sobre el significado de la RSU. De otra forma, la RSU queda en mera filantropía o en acciones bonitas y deseables, pero esporádicas y sin continuidad. Esto concuerda con la percepción de que la Facultad no es socialmente responsable, como indicaron los alumnos y reportaron Collí y Cano (2014).

Los autores esperaban que los profesores, al ser profesionales de prestigio en el medio, también tuvieran mayor orientación hacia la práctica de la RSU. Sin embargo, son competentes en lo que hacen pero desconocen el concepto de RSE y de RSU en el sentido amplio. No basta con ser un experto en el campo del conocimiento para serlo en la RSU, pero el comportamiento socialmente responsable sí implica ser un experto en el conocimiento, por lo que dicho comportamiento debe tomarse en cuenta desde el perfil de contratación de los profesores. Los alumnos admiran a sus profesores por lo que éstos saben y por su prestigio profesional, pero no les exigen un comportamiento socialmente responsable porque los alumnos mismos desconocen el concepto de RSU. Esto significa que si esos profesores, aprovechando que captan la atención del alumno, adoptaran y promovieran la RSU y que lo hicieran con entusiasmo, los alumnos les seguirían. Desde este punto de vista la RSU es clave del desarrollo sostenible de la Institución, como indican Rochlin (2005) y Barroso (2008).

En la tabla 2 se muestran los factores críticos a considerar, desde las respuestas de los alumnos, para implementar la RSU en la Facultad, de los cuales el más crítico es el compromiso y participación de sus autoridades, profesores y del personal de la institución en acciones y proyectos de RSU. También fueron mencionados en la Fase 1 y por los administrativos, lo que indica que las percepciones de directivos y administradores se ven corroborados por los factores principales provenientes de las respuestas de los alumnos. Si no se comienza desde dentro de la institución, los alumnos no se van a involucrar en la RSU y la tomarán como un conjunto de acciones para aprobar una materia o para lograr activismo político dentro de la institución.

Es necesaria la vinculación con otras instituciones, pero dicha vinculación puede comenzar también con otras escuelas y facultades de la misma universidad. Estar bien con el gobierno es importante, pero lo es más el ejemplo del personal universitario para fomentar la RSU. Es poner al ser humano por encima de la política y poner la política al servicio del mejoramiento de la calidad de vida del ser humano. Así, puede decirse que una auténtica vivencia de la visión y misión por 
parte del personal y alumnos es el primer paso para el comportamiento socialmente responsable, como propone Medina (2006).

El estudiante espera más acciones para el cuidado ambiental y la mejoría de la relación con la comunidad. Sin embargo, como se observa en los resultados de la fase 1 , hay personal reacio a colaborar en estas acciones probablemente porque no perciben que la RSU es importante (Rochlin, 2005) o porque falta capacidad negociadora por parte de las autoridades de la institución. Ayudaría el que la participación se convocara vía reglamento, a todos sus profesores y personal, con la debida relación con el sindicato, pero hay que recordar que la RSU es un valor, por lo que se enseña con el ejemplo más que en clases o con sanciones. Se espera que sea practicada por todo el personal y alumnos, porque es algo que vale la pena y permite a la institución ser más competitiva, respetando la dignidad de las personas, comunidades y medio ambiente, como establecen Sánchez et al. (2007). El mejor reglamento, si no se practica, es estéril y solo serviría para obtener una acreditación o certificación, pero para nada más, lo que refuerza la importancia de practicar la visión y la misión, puesto que si solamente se enseña conocimiento pero no se inculcan valores para la vida, solo está capacitando al alumno para la vida laboral, pero no le está formando adecuadamente.

Es necesario que los alumnos vean a sus profesores comprometidos y satisfechos con lo que hacen, que haya un ambiente agradable entre el personal de la Facultad y que sus autoridades y profesores sean los primeros en participar en acciones socialmente responsables no como un deber a cumplir, sino como una oportunidad para contribuir con el mejoramiento del medio y de la sociedad.

\section{CONCLUSIONES}

Los factores para implementar exitosamente la RSU son el compromiso y participación del personal de la institución; las acciones conjuntas con otras instituciones; énfasis en el cuidado ambiental; participación de la Institución en actividades sociales; procurar la satisfacción de los usuarios de la Institución; apreciar la importancia de la institución para el desarrollo, así como mantener y mejorar las relaciones con el Gobierno. Sin embargo, también deben considerarse la habilidad para negociar de las autoridades con los profesores, sindicato y alumnos, así como hacer públicos los valores, visión y misión de la Institución. Por lo expuesto este trabajo, dichos factores pueden ser aplicados en otras universidades públicas, aunque también en las privadas con los cambios correspondientes según su orientación y estructura.

Las universidades, en lugar de ser consideradas como centros de enseñanza, deben verse como lugares en que el talento y la oportunidad se encuentren para el desarrollo del ser humano y la sociedad, para lo que es necesario realizar modificaciones tanto en los programas académicos como en actividades vivenciales. Entonces, no basta con contratar profesores que sean grandes profesionistas desde el punto de vista técnico, sino que hay que lograr que sean socialmente responsables y que logren transmitir esto a sus alumnos, ya que la RSU sigue a uno a todas partes.

\section{Recomendaciones}

Con base en lo anterior, se recomienda tener una sesión al semestre en la que se reflexione en toda la Facultad acerca de la visión y la misión de la misma, o solamente la misión. Puede establecerse el «Día de la misión», en que se hagan actividades al respecto, mesas de discusión, paneles de expertos, concursos de carteles o de ensayos sobre cómo cumplir la misión, en que participen alumnos y profesores. El objetivo es orientar a la Facultad a mejores prácticas docentes y de RSU.

A los profesores se les pueden impartir cursos sobre RSE y cómo transformarla en RSU más allá de pensar en visitas a albergues o solo con cumplir bien sus tareas. Ellos, aunque no se den cuenta a veces, son ejemplos vivos para los alumnos y la imagen que la Facultad tenga ante los educandos. El que la participación en acciones de RSE se tome en cuenta en la evaluación institucional y en la asignación de bonos, estímulos y promociones sería muy recomendable, aunque conforme pase el tiempo y se conforme la cultura de RSU, se logre la participación con base en el desarrollo de la voluntad y el compromiso. 


\section{REFERENCIAS}

Barroso, F. (2008, septiembre-diciembre). La responsabilidad social organizacional. Un estudio en cuarenta organizaciones de la ciudad de Mérida, Yucatán. Contaduría y Administración, 226, 73-91.

Collí, K. y Cano, L. (2014). Implementación de la responsabilidad social universitaria en la Universidad Autónoma de Campeche. Manuscrito no publicado. México: Universidad Anáhuac Mayab.

Chomsky, N. (2003). Los límites de la globalización. Barcelona: Ariel.

De la Cruz, C. (2010). La responsabilidad de la universidad en la sociedad que la acoge: ¿complementariedad o antagonismo? En M. De la Cuesta, C. De la Cruz y J. M. Rodríguez (Eds.). España: Netbiblio.

De la Cruz, C. (2011). Reflexiones sobre la permanencia de la responsabilidad en las instituciones de educación superior; retos, limitaciones y oportunidades. En C. H. Forero (Ed.). Responsabilidad social universitaria. El pensamiento universitario, 21. Bogotá, Colombia, Asociación Colombiana de Universidades.

Kliksberg, B. (2009, febrero). Los desafíos éticos en un mundo paradojal: el rol de la universidad. Reforma y Democracia.

Medina, L.M. (2006, mayo). La responsabilidad social de la organización. Ponencia presentada en el $X$ Congreso Anual de la Academia de Ciencias Administrativas A.C. (ACACIA). Universidad Autónoma de San Luis Potosí. San Luis Potosí.

Porto, N. y Castromán, J. (2006, septiembrediciembre). Responsabilidad social: un análisis de la situación actual en México y España. Contaduría y Administración, 220, 67-87.
Ramos, H. (2006, primer semestre). Ética y responsabilidad social. Reflexiones y perspectiva sistémica. The Anáhuac Journal, 6(1), 56-71.

Rochlin, S. (2005, agosto). Llevar la responsabilidad corporativa al ADN de su organización. Harvard Business Review, 83(8), 31-38.

Rojas, M.; M'Zali, B.; Turcotte, M. y Kooli, M. (2006). Corporate social responsibility, the stakeholder approach and beyond: in search of theoretical explanations for 'doing well while doing good's. Ponencia presentada en el $X$ Congreso Anual de la Academia de Ciencias Administrativas A.C. (ACACIA). Universidad Autónoma de San Luis Potosí, San Luis Potosí.

Sánchez, L. A.; Placencia, M. y Pedroza, A. (2007). Diagnóstico exploratorio del nivel de responsabilidad social organizacional percibido por las organizaciones involucradas en el bioclúster de occidente. Ponencia presentada en el XI Congreso Anual de la Academia de Ciencias Administrativas A.C. (ACACIA). Instituto Tecnológico y de Estudios Superiores de Oriente (ITESO), Guadalajara, Jalisco.

Somoggi, L., (2005, agosto). Más que una estrategia, una filosofía. Harvard Business Review, 83(8), 1728.

Valverde J. (2011). Responsabilidad social universitaria: una mirada desde Costa Rica. Eecuperado el 14 de Julio de 2014 de http:// w w w. i e s a I c. un e s co.org. ve / index.php?option=com_content\&view=article\&id= 2597\%3Aresponsabilidad-social-universitariaun a-mirada-desde-costa-rica\&catid $=126 \% 3$ Anoticias-paginanueva\&ltemid $=712 \&$ lang $=$ es

Vallaeys, F., De la Cruz, C. y Sasia, P. (2009). Responsabilidad Social Universitaria. Manual primeros pasos, México D.F.: Mc Graw Hill. 